\title{
Nanostructured Thermoset / Thermoset Blends Compatibilized with an Amphiphilic Block Copolymer
}

\author{
Brian J. Rohde, ${ }^{a}$ Tyler E. Culp, ${ }^{b}$ Enrique D. Gomez, ${ }^{b}$ Jan Ilavsky, ${ }^{c}$ Ramanan \\ Krishnamoorti, ${ }^{a} *$ Megan L. Robertson ${ }^{a, *}$ \\ aDepartment of Chemical and Biomolecular Engineering, University of Houston \\ ${ }^{b}$ Department of Chemical Engineering and the Materials Research Institute, The Pennsylvania \\ State University \\ ${ }^{\mathrm{c}}$ Advanced Photon Source, Argonne National Laboratory, 9700 S Cass Avenue, Argonne, IL, \\ USA.
}

*Corresponding authors

4726 Calhoun Road

S222 Engineering Building 1

University of Houston

Houston, TX 77204-4004

Krishnamoorti: ramanan@uh.edu, Robertson: mlrobertson@uh.edu

Krishnamoorti: 713-743-4307, Robertson: 713-743-2748 


\section{BLEND NOMENCLATURE}

Blends of epoxy resin and PDCPD are labeled as xxE-y.y, where $\mathrm{xx}$ corresponds to the volume \% of epoxy resin (consisting of DGEBA, NMA, and K54), y.y corresponds to the weight $\%$ of block copolymer The weight $\%$ of block copolymer addition is determined from the mass of the uncompatibilized blend of epoxy resin and DCPD. For example, in blend 30E-5.0 is a 30:70 (volume ratio) mixture of epoxy resin : PDCPD, and the blend contained with $5.0 \mathrm{wt} \%$ PB-PEO

\section{TABLE OF CONTENTS}

\section{Section 1: Poly(1,4-butadiene- $b$-ethylene oxide) (PB-PEO) Block Copolymer} Characterization

Figure S1: NMR

Figure S2: GPC

Figure S3: DSC

\section{Section 2: X-ray Scattering Data Obtained from Fully Cured Blends}

Figure S4: Blend 100E-5.0 (Rigaku instrument)

Figure S5: 50E blends at various wt\% BCP

Figure S6: Blends containing $5 \mathrm{wt} \% \mathrm{BCP}$ at various epoxy resin : DCPD volume ratios

Table S1: Derived scattering parameters for 50E blends w/ varying BCP wt\%

Table S2: Derived scattering parameters for epoxy-rich asymmetric blends

Table S3: Derived scattering parameters for PDCPD-rich asymmetric blends

\section{Section 3: Differential Scanning Calorimetry Data from Fully Cured Blends}

Figure S7: Blends 100E-5.0 and 0E-5.0

\section{Section 4: Mechanical Properties of Uncompatibilized and Compatibilized Blends.}

Table S4: Mechanical Properties of Blends

\section{Section 5: Critical Stress Intensity Calculations}

Table S5: Testing parameters for SENB specimens. 


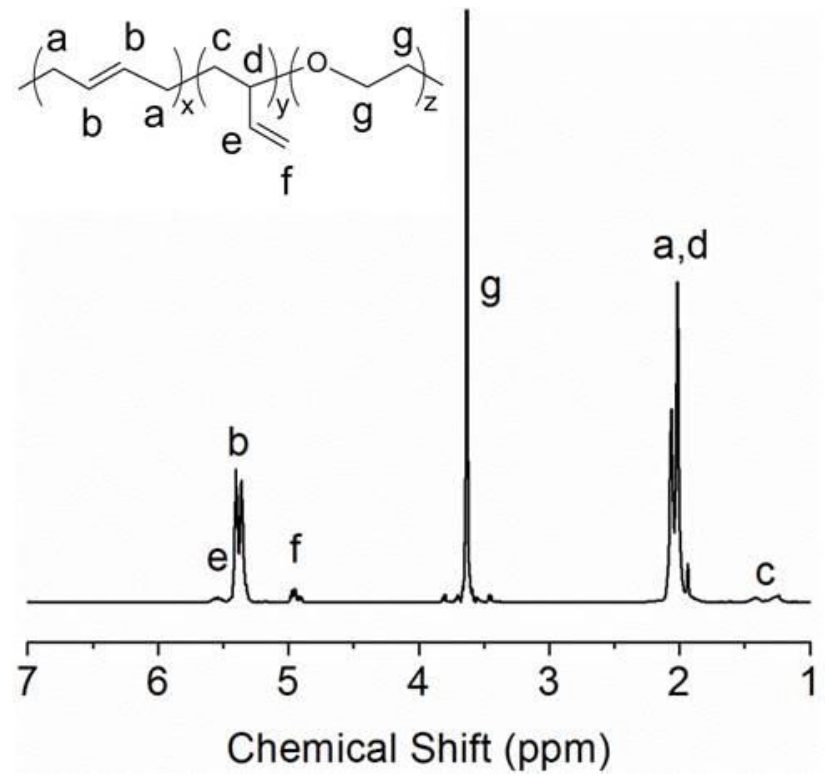

Figure S1: ${ }^{1} \mathrm{HNMR}$ spectrum and peak assignment of PB-PEO

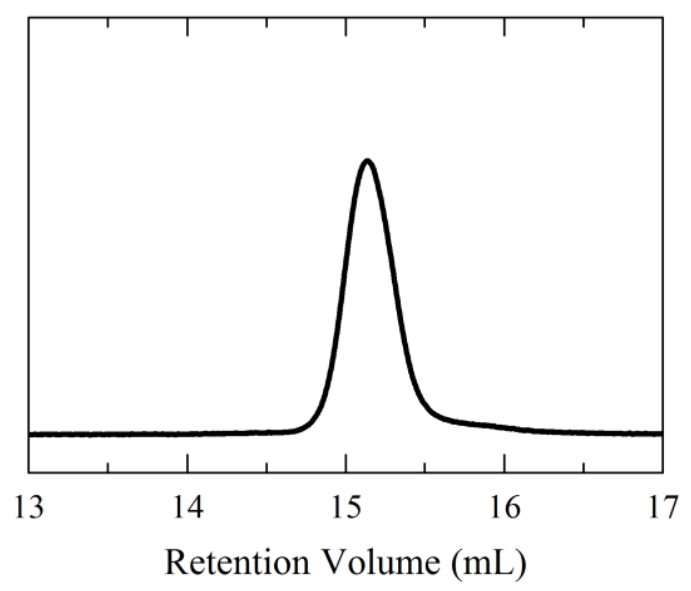

Figure S2: GPC data obtained from PB-PEO 


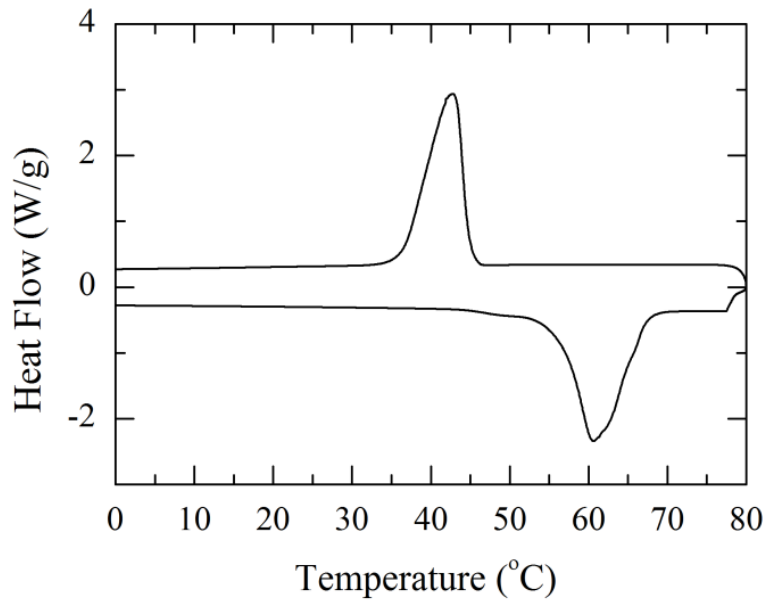

Figure S3: Heat flow obtained from PB-PEO as determined by DSC showing the crystallization and melting of the $\mathrm{PEO}$ domain (exotherm up). The $\mathrm{Tg}$ of the $\mathrm{PB}$ block was below $-90^{\circ} \mathrm{C}$ and not recorded. 
Section 2: Ultra-small and small angle X-ray scattering (USAXS and SAXS) of Fully Cured Blends

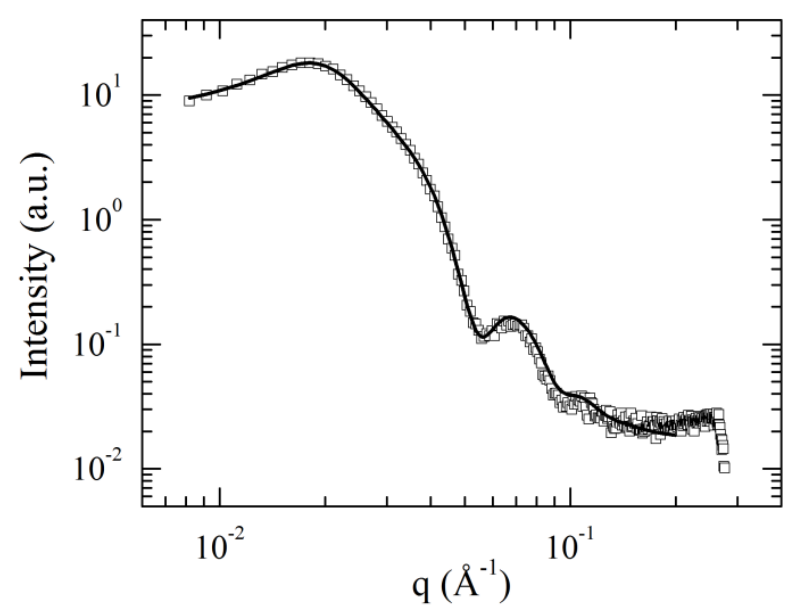

Figure S4: SAXS data of 100E-5.0 from a benchtop Rigaku SAXS instrument on an arbitrary scale. Error bars are smaller than the data point markers. Solid line represent a spherical fit with hard-sphere interactions.

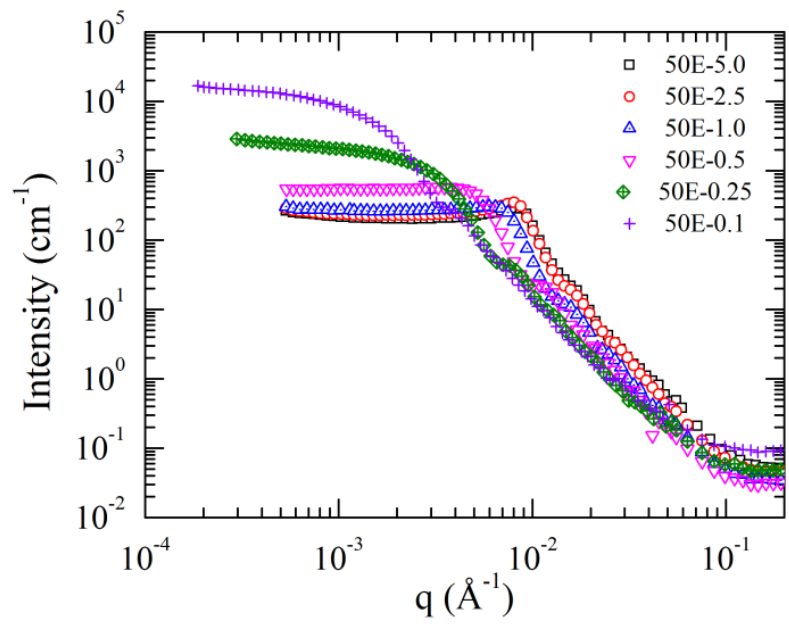

Figure S5: Reduced USAXS data on the absolute scale for all 50E blends. 


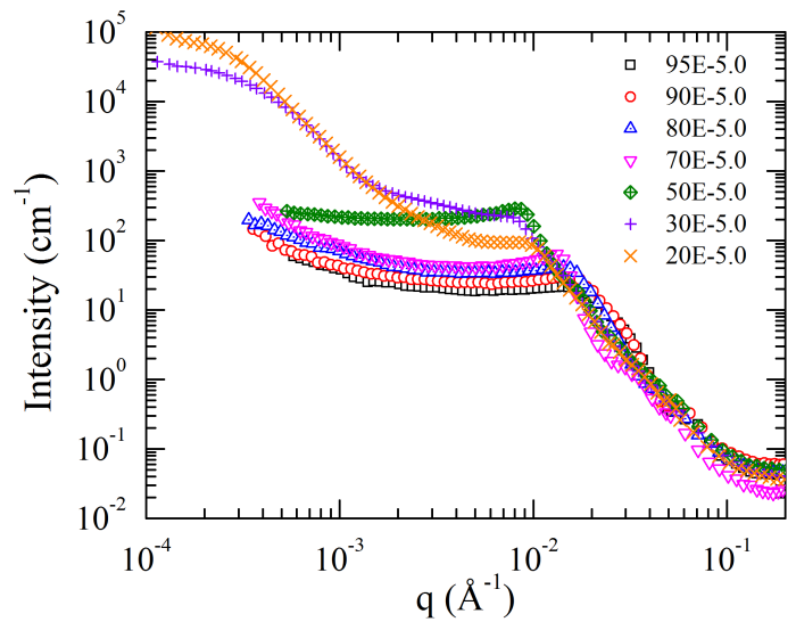

Figure S6: Reduced USAXS data on the absolute scale for all asymmetric blends.

Table S1: Form factor and structure factor fitting parameters for 50E blends containing block copolymer. Standard deviation values of the average micelle radii represent the widths of the applied sphere radius Gaussian distribution.

\begin{tabular}{|c|c|c|c|c|}
\hline Blend & $\begin{array}{c}\text { Block } \\
\text { Copolymer } \\
\text { wt \% }\end{array}$ & $\begin{array}{c}\text { Average Micelle } \\
\text { Radius } \\
(\mathbf{n m})\end{array}$ & $\begin{array}{c}\text { Inter-sphere } \\
\text { Distance } \\
(\mathbf{n m})\end{array}$ & $\begin{array}{c}\text { Sphere Volume } \\
\text { Fraction }\end{array}$ \\
\hline $50 \mathrm{E}-5.0$ & 5 & $24 \pm 6$ & 35 & 0.40 \\
\hline $50 \mathrm{E}-2.5$ & 2.5 & $26 \pm 5$ & 37 & 0.40 \\
\hline $50 \mathrm{E}-1.0$ & 1 & $32 \pm 4$ & 40 & 0.31 \\
\hline $50 \mathrm{E}-0.5$ & 0.5 & $45 \pm 6$ & 51 & 0.24 \\
\hline $50 \mathrm{E}-0.25$ & 0.25 & $65 \pm 10$ & -- & -- \\
\hline $50 \mathrm{E}-0.1$ & 0.1 & $130 \pm 20$ & -- & - \\
\hline
\end{tabular}

Table S2: Form factor and structure factor fitting parameters for epoxy rich asymmetric blends containing $5.0 \mathrm{wt} \%$ block copolymer. Standard deviation values of the average micelle radii represent the widths of the applied sphere radius Gaussian distribution.

\begin{tabular}{|c|c|c|c|c|}
\hline Blend & $\begin{array}{c}\text { Epoxy } \\
\text { Resin } \\
\text { Vol \% }\end{array}$ & $\begin{array}{c}\text { Average Micelle } \\
\text { Radius } \\
\text { (nm) }\end{array}$ & $\begin{array}{c}\text { Inter-sphere } \\
\text { Distance } \\
(\mathbf{n m})\end{array}$ & $\begin{array}{c}\text { Sphere Volume } \\
\text { Fraction }\end{array}$ \\
\hline $100 \mathrm{E}-5.0$ & 100 & $8.2 \pm 0.9$ & 14.8 & 0.17 \\
\hline $95 \mathrm{E}-5.0$ & 95 & $8.6 \pm 1.2$ & 16.4 & 0.21 \\
\hline $90 \mathrm{E}-5.0$ & 90 & $9.4 \pm 1.3$ & 17.5 & 0.28 \\
\hline $80 \mathrm{E}-5.0$ & 80 & $12 \pm 2$ & 18.3 & 0.30 \\
\hline $70 \mathrm{E}-5.0$ & 70 & $17 \pm 2$ & 23.1 & 0.42 \\
\hline
\end{tabular}


Table S3: Form factor and structure factor fitting parameters for DCPD rich asymmetric blends containing $5.0 \mathrm{wt} \%$ block copolymer

\begin{tabular}{|c|c|c|c|c|c|c|}
\hline Blend & $\begin{array}{c}\text { Epoxy } \\
\text { Resin } \\
\text { Vol \% }\end{array}$ & $\begin{array}{c}\text { Micelle } \\
\text { Radius } \\
(\mathbf{n m})^{\mathbf{a}}\end{array}$ & $\begin{array}{c}\text { Inter-sphere } \\
\text { Distance } \\
(\mathbf{n m})\end{array}$ & $\begin{array}{c}\text { Sphere } \\
\text { Volume } \\
\text { Fraction }\end{array}$ & $\begin{array}{c}\mathbf{R}_{\mathbf{g}} \\
(\mathbf{n m})^{\mathbf{b}}\end{array}$ & $\mathbf{P}$ \\
\hline $20 \mathrm{E}-5.0$ & 20 & $18 \pm 6$ & 29 & 0.27 & 600 & 3.8 \\
\hline $30 \mathrm{E}-5.0$ & 30 & $22 \pm 7$ & 36 & 0.28 & 420 & 4 \\
\hline
\end{tabular}

${ }^{a}$ Average and standard deviation of the micelle radii represent. The standard deviation represents the width of the applied sphere radius Gaussian distribution.

${ }^{\mathrm{b}}$ Values in parentheses represent the unified fit applied the intermediate-q region on 30E-5.0.

\section{Section 3: Differential Scanning Calorimetry of Fully Cured Blends}

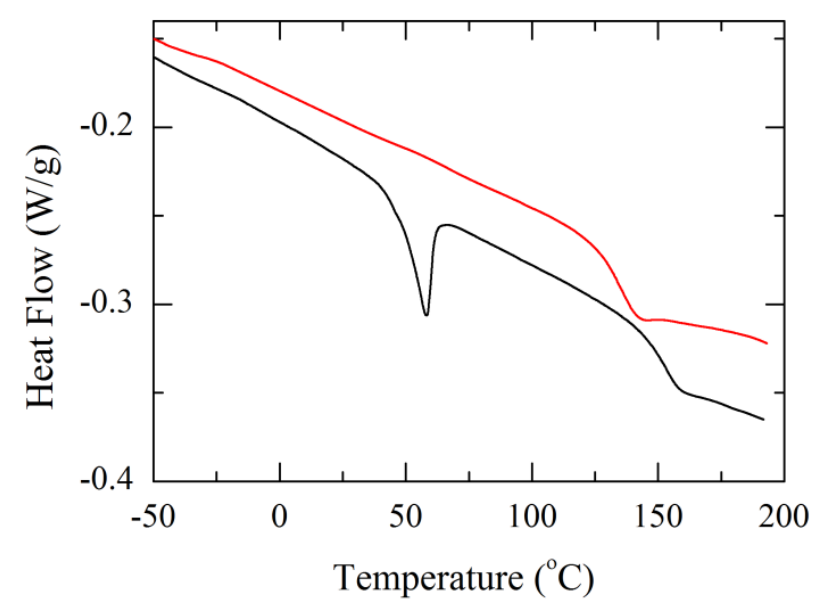

Figure S7: Truncated DSC heat flow curve of blends 100E-5.0 (5 wt $\%$ BCP in fully cured neat epoxy resin, top red line) and 0E-5.0 (5 wt $\%$ BCP in fully cured neat PDCPD, bottom black line). No crystallization or melting was apparent in the $100 \mathrm{E}-5.0\left(-90^{\circ} \mathrm{C}\right.$ to $\left.200^{\circ} \mathrm{C}\right)$, while there was a single $\mathrm{Tg}_{\mathrm{g}}$ at $135^{\circ} \mathrm{C}$. For the $0 \mathrm{E}-5.0$ sample, crystallization and melting was apparent at $-24^{\circ} \mathrm{C}$ and $58^{\circ} \mathrm{C}$, respectively. A single $\mathrm{Tg}$ was observable in the $0 \mathrm{E}-5.0$ sample at $153^{\circ} \mathrm{C}$. 
Section 4: Mechanical Properties of Uncompatibilized and Compatibilized Blends.

Table S4: Tabulated fracture and tensile properties for select uncompatibilized blends and compatibilized blends containing 0.1 and $0.5 \mathrm{wt} \%$ PB-PEO. Data for the uncompatibilized blends is from prior publications. ${ }^{43}$

\begin{tabular}{|c|c|c|c|c|c|}
\hline Blend & $\begin{array}{c}\text { Epoxy } \\
\text { Resin } \\
\text { Vol \% }\end{array}$ & $\begin{array}{c}\text { PB-PEO } \\
\text { Wt \% }\end{array}$ & $\begin{array}{c}\text { Tensile } \\
\text { Strength } \\
\mathbf{( M P a )}\end{array}$ & $\begin{array}{c}\text { Modulus } \\
(\mathbf{G P a})\end{array}$ & $\begin{array}{c}\mathbf{K}_{\mathbf{I C}} \\
\left(\mathbf{M P a}^{*} \mathbf{m}^{\mathbf{1} \mathbf{2}} \mathbf{)}\right.\end{array}$ \\
\hline $100 \mathrm{E}$ & 100 & 0 & $91+/-8$ & $2.62+/-0.10$ & $.58+/-0.09$ \\
\hline $100 \mathrm{E}-0.1$ & 100 & 0.1 & $87+/-8$ & $2.72+/-0.08$ & $.58+/-0.04$ \\
\hline $100 \mathrm{E}-0.5$ & 100 & 0.5 & $94+/-3$ & $2.68+/-0.05$ & $.60+/-0.08$ \\
\hline $90 \mathrm{E}$ & 90 & 0 & $100+/-4$ & $2.58+/-0.05$ & $0.70+/-0.02$ \\
\hline $90 \mathrm{E}-0.1$ & 90 & 0.1 & $84+/-4$ & $2.57+/-0.12$ & $0.71+/-0.03$ \\
\hline $90 \mathrm{E}-0.5$ & 90 & 0.5 & $86+/-3$ & $2.66+/-0.03$ & $0.68+/-0.03$ \\
\hline $70 \mathrm{E}$ & 70 & 0 & $79+/-2$ & $2.54+/-0.05$ & $1.25+/-0.10$ \\
\hline $70 \mathrm{E}-0.1$ & 70 & 0.1 & $81+/-2$ & $2.60+/-0.04$ & $1.57+/-0.08$ \\
\hline $70 \mathrm{E}-0.5$ & 70 & 0.5 & $85+/-1$ & $2.38+/-0.05$ & $1.37+/-0.07$ \\
\hline $50 \mathrm{E}$ & 50 & 0 & $76+/-1$ & $2.26+/-0.04$ & $1.51+/-0.07$ \\
\hline $50 \mathrm{E}-0.1$ & 50 & 0.1 & $77+/-1$ & $2.28+/-0.04$ & $1.82+/-0.04$ \\
\hline $50 \mathrm{E}-0.5$ & 50 & 0.5 & $75+/-2$ & $2.29+/-0.14$ & $1.69+/-0.08$ \\
\hline
\end{tabular}

\section{Section 5: Critical Stress Intensity Calculations}

The critical stress intensity factor, KIC, determined from ASTM standard D5045 is defined as (assuming a valid test)

$$
K_{I C}=\left(\frac{P_{Q}}{B W^{1 / 2}}\right) f(x)
$$

where for $0<\mathrm{x}<1$,

$$
\begin{gathered}
f(x)=6 x^{1 / 2} \frac{\left[1.99-x(1-x)\left(2.15-3.93 x+2.7 x^{2}\right)\right]}{(1+2 x)(1-x)^{3 / 2}} \\
\left.P_{Q}=\text { The peak load determined from testing (see Figure } \mathrm{S} 22\right), \mathrm{kN} \\
B=\text { specimen thickness, } \mathrm{cm}
\end{gathered}
$$




$$
\begin{gathered}
\qquad \begin{array}{c}
W=\text { specimen width, } \mathrm{cm} \\
\left.a_{e f f}=\text { effective crack length (average of the length both sides } \mathrm{a}_{1} \text { and } \mathrm{a}_{2}\right), \mathrm{cm} \\
x=\frac{a_{\text {eff }}}{W}
\end{array}
\end{gathered}
$$

The peak load, $P_{Q}$, is determined from analysis of the load $(\mathrm{kN})$ versus deflection plot as shown in Figure S8, where a line following the slope of the linear region is determined ( - in Figure S8) to establish the initial compliance and used to create a secondary compliance line (- - in Figure S8) with a slope that is 0.953 of the initial compliance slope. The max load, $\mathrm{P}_{\mathrm{Max}}$, differs from $\mathrm{P}_{\mathrm{Q}}$ and represents the maximum in the raw data. The intersection of the secondary compliance (- - ) and the raw data is the peak load, $P_{Q}$. If $P_{\operatorname{Max}}<\mathrm{PQ}_{\mathrm{Q}}, P_{\operatorname{Max}}$ is used as $P_{Q}$ in the calculation of KIC. If $P_{M a x} / P_{Q}<1.1, P_{Q}$ in the calculation of $\mathrm{K}_{\mathrm{IC}}$. If $P_{\operatorname{Max}} / P_{Q}>1.1$, the test is not valid.

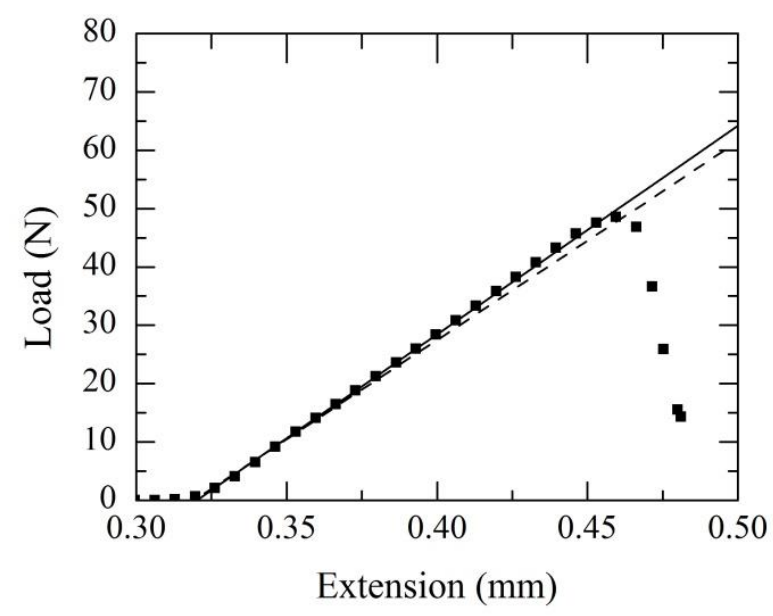

Figure S8: Raw load versus displacement data for a neat epoxy SENB specimen. The solid black line (-) is the initial compliance used to determine the secondary compliance with $5 \%$ great compliance than the initial (- - ). The maximum in the raw data is PMax and the intersection of the raw data and the secondary compliance is $\mathrm{P}_{\mathrm{Q}}$.

In order to maintain the validity of the testing, the samples must satisfy the plane strain criteria expresses by 


$$
B, a,(W-a)>2.5\left(\frac{K_{I C}}{\sigma_{y}}\right)^{2}
$$

where $\mathrm{K}_{\mathrm{IC}}$ is the critical stress intensity factor determined above and $\sigma_{y}$ is the yield stress of the material determined from tensile testing. Should this criteria not be satisfied (i.e., the lefthand side needs to be greater than the right), plane strain does not occur and the test is not valid. All samples satisfied this criterion. All relevant fracture testing parameters are included in Table S5. 
Table S5: Testing parameters for SENB specimens of compatibilized blends.

\begin{tabular}{|c|c|c|c|c|c|c|c|c|c|c|c|c|}
\hline 50E-0.1 & $\begin{array}{l}\text { Width } \\
\text { (cm) }\end{array}$ & $\begin{array}{l}\text { Thick } \\
\text {-ness } \\
\text { (cm) }\end{array}$ & $\begin{array}{l}\text { Length } \\
\text { (cm) }\end{array}$ & $\mathbf{a}_{1}$ & $\mathbf{a}_{2}$ & aeff & W-a & $\sigma_{\mathrm{y}}$ & $\mathbf{x}$ & $\mathbf{P}_{\text {Max }}$ & $\mathbf{f}(\mathbf{x})$ & $\begin{array}{l}\mathrm{K}_{\mathrm{Q}} \\
(\mathrm{MPa}- \\
\left.\mathbf{m}^{\mathbf{1 / 2}}\right)\end{array}$ \\
\hline 1 & 1.198 & 0.603 & 5.372 & 0.398 & 0.398 & 0.398 & 0.8 & 90 & 0.332 & 0.177 & 6.61 & 1.77 \\
\hline 2 & 1.194 & 0.623 & 5.356 & 0.639 & 0.669 & 0.654 & 0.54 & 91 & 0.548 & 0.102 & 12.47 & 1.87 \\
\hline 3 & 1.21 & 0.622 & 5.362 & 0.508 & 0.525 & 0.5165 & 0.6935 & 90 & 0.427 & 0.1475 & 8.55 & 1.84 \\
\hline 4 & 1.224 & 0.605 & 5.343 & 0.672 & 0.672 & 0.672 & 0.552 & 91 & 0.549 & 0.099 & 12.53 & 1.85 \\
\hline 5 & 1.21 & 0.625 & 5.356 & 0.386 & 0.438 & 0.412 & 0.798 & 92 & 0.340 & 0.181 & 6.76 & 1.78 \\
\hline \multicolumn{13}{|l|}{ 50E-0.5 } \\
\hline 1 & 1.28 & 0.605 & 5.38 & 0.38 & 0.386 & 0.383 & 0.897 & 90 & 0.299 & 0.199 & 6.07 & 1.77 \\
\hline 2 & 1.3 & 0.601 & 5.365 & 0.565 & 0.475 & 0.52 & 0.78 & 91 & 0.400 & 0.15 & 7.93 & 1.74 \\
\hline 3 & 1.28 & 0.609 & 5.36 & 0.824 & 0.72 & 0.772 & 0.508 & 90 & 0.603 & 0.07 & 15.27 & 1.55 \\
\hline 4 & 1.299 & 0.609 & 5.36 & 0.614 & 0.509 & 0.5615 & 0.7375 & 90 & 0.432 & 0.134 & 8.68 & 1.68 \\
\hline 5 & 1.287 & 0.602 & 5.343 & 0.535 & 0.431 & 0.483 & 0.804 & 91 & 0.375 & 0.157 & 7.41 & 1.70 \\
\hline \multicolumn{13}{|l|}{ 70E-0.1 } \\
\hline 1 & 1.218 & 0.62 & 5.373 & 0.664 & 0.604 & 0.634 & 0.584 & 90 & 0.521 & 0.092 & 11.38 & 1.53 \\
\hline 2 & 1.216 & 0.619 & 5.377 & 0.708 & 0.714 & 0.711 & 0.505 & 91 & 0.585 & 0.071 & 14.24 & 1.48 \\
\hline 3 & 1.224 & 0.623 & 5.374 & 0.579 & 0.64 & 0.6095 & 0.6145 & 90 & 0.498 & 0.11 & 10.58 & 1.69 \\
\hline 4 & 1.208 & 0.614 & 5.372 & 0.78 & 0.741 & 0.7605 & 0.4475 & 90 & 0.630 & 0.063 & 16.97 & 1.58 \\
\hline 5 & 1.221 & 0.606 & 5.375 & 0.497 & 0.586 & 0.5415 & 0.6795 & 91 & 0.443 & 0.118 & 8.97 & 1.58 \\
\hline \multicolumn{13}{|l|}{ 70E-0.5 } \\
\hline 1 & 1.201 & 0.608 & 5.358 & 0.501 & 0.507 & 0.504 & 0.697 & 90 & 0.420 & 0.107 & 8.38 & 1.34 \\
\hline 2 & 1.257 & 0.606 & 5.358 & 0.703 & 0.746 & 0.7245 & 0.5325 & 91 & 0.576 & 0.074 & 13.81 & 1.50 \\
\hline 3 & 1.215 & 0.611 & 5.358 & 0.471 & 0.405 & 0.438 & 0.777 & 90 & 0.360 & 0.126 & 7.12 & 1.33 \\
\hline 4 & 1.226 & 0.608 & 5.359 & 0.461 & 0.51 & 0.4855 & 0.7405 & 90 & 0.396 & 0.118 & 7.84 & 1.37 \\
\hline 5 & 1.219 & 0.605 & 5.359 & 0.561 & 0.594 & 0.5775 & 0.6415 & 91 & 0.474 & 0.089 & 9.82 & 1.31 \\
\hline 6 & 1.215 & 0.611 & 5.365 & 0.57 & 0.531 & 0.5505 & 0.6645 & 92 & 0.453 & 0.101 & 9.23 & 1.38 \\
\hline \multicolumn{13}{|l|}{ 90E-0.1 } \\
\hline 1 & 1.254 & 0.609 & 5.362 & 0.681 & 0.62 & 0.6505 & 0.6035 & 90 & 0.519 & 0.0433 & 11.31 & 0.72 \\
\hline 2 & 1.214 & 0.607 & 5.357 & 0.528 & 0.528 & 0.528 & 0.686 & 91 & 0.435 & 0.057 & 8.75 & 0.75 \\
\hline 3 & 1.218 & 0.608 & 5.36 & 0.613 & 0.68 & 0.6465 & 0.5715 & 90 & 0.531 & 0.0399 & 11.77 & 0.70 \\
\hline 4 & 1.228 & 0.61 & 5.364 & 0.754 & 0.646 & 0.7 & 0.528 & 90 & 0.570 & 0.0352 & 13.49 & 0.70 \\
\hline 5 & 1.255 & 0.608 & 5.361 & 0.573 & 0.726 & 0.6495 & 0.6055 & 91 & 0.518 & 0.0407 & 11.27 & 0.67 \\
\hline \multicolumn{13}{|l|}{ 90E-0.5 } \\
\hline 1 & 1.29 & 0.608 & 5.356 & 0.631 & 0.591 & 0.611 & 0.679 & 90 & 0.474 & 0.0509 & 9.81 & 0.72 \\
\hline 2 & 1.291 & 0.608 & 5.365 & 0.648 & 0.764 & 0.706 & 0.585 & 91 & 0.547 & 0.0359 & 12.43 & 0.65 \\
\hline 3 & 1.29 & 0.611 & 5.356 & 0.722 & 0.596 & 0.659 & 0.631 & 90 & 0.511 & 0.0418 & 11.03 & 0.66 \\
\hline 4 & 1.286 & 0.609 & 5.361 & 0.431 & 0.502 & 0.4665 & 0.8195 & 90 & 0.363 & 0.0674 & 7.17 & 0.70 \\
\hline \multicolumn{13}{|l|}{ 100E-0.1 } \\
\hline 1 & 1.201 & 0.605 & 5.359 & 0.488 & 0.446 & 0.467 & 0.734 & 90 & 0.389 & 0.0527 & 7.69 & 0.61 \\
\hline 2 & 1.201 & 0.607 & 5.355 & 0.42 & 0.497 & 0.4585 & 0.7425 & 91 & 0.382 & 0.0475 & 7.54 & 0.54 \\
\hline 3 & 1.248 & 0.606 & 5.362 & 0.458 & 0.458 & 0.458 & 0.79 & 90 & 0.367 & 0.0563 & 7.25 & 0.60 \\
\hline
\end{tabular}




\begin{tabular}{l|l|l|l|l|l|l|l|l|l|l|l|l}
\hline $\mathbf{4}$ & 1.213 & 0.607 & 5.359 & 0.513 & 0.575 & 0.544 & 0.669 & 90 & 0.448 & 0.044 & 9.10 & 0.60 \\
\hline $\mathbf{5}$ & 1.216 & 0.605 & 5.359 & 0.703 & 0.791 & 0.747 & 0.469 & 91 & 0.614 & 0.0224 & 15.96 & 0.54 \\
\hline $\mathbf{5}$ & 1.229 & 0.606 & 5.359 & 0.616 & 0.622 & 0.619 & 0.61 & 92 & 0.504 & 0.0379 & 10.77 & 0.61 \\
\hline $\mathbf{1 0 0 E - 0 . 5}$ & & & & & & & & & & & \\
\hline $\mathbf{1}$ & 1.278 & 0.61 & 5.365 & 0.671 & 0.615 & 0.643 & 0.635 & 90 & 0.503 & 0.0344 & 10.76 & 0.54 \\
\hline $\mathbf{2}$ & 1.302 & 0.611 & 5.373 & 0.62 & 0.613 & 0.6165 & 0.6855 & 91 & 0.474 & 0.0467 & 9.81 & 0.66 \\
\hline $\mathbf{3}$ & 1.293 & 0.61 & 5.358 & 0.535 & 0.603 & 0.569 & 0.724 & 90 & 0.440 & 0.0425 & 8.88 & 0.54 \\
\hline $\mathbf{4}$ & 1.294 & 0.609 & 5.361 & 0.733 & 0.721 & 0.727 & 0.567 & 90 & 0.562 & 0.0324 & 13.10 & 0.61 \\
\hline $\mathbf{5}$ & 1.337 & 0.606 & 5.368 & 0.664 & 0.65 & 0.657 & 0.68 & 91 & 0.491 & 0.045 & 10.37 & 0.67 \\
\hline
\end{tabular}

